\title{
Measurable residual disease assessed by mass spectrometry in peripheral blood in multiple myeloma in a phase II trial of carfilzomib, lenalidomide, dexamethasone and autologous stem cell transplantation
}

\author{
Benjamin A. Derman (1)', Andrew T. Stefka (10 ${ }^{1}$, Ken Jiang ${ }^{1}$, Amanda Mclver ${ }^{1}$, Tadeusz Kubicki $\mathbb{C}^{2}$, Jagoda K. Jasielec ${ }^{1}$ and \\ Andrzej J. Jakubowiak (1)
}

Dear Editor,

Despite advances in the detection of measurable (minimal) residual disease (MRD) in multiple myeloma (MM), some patients who are MRD negative by highly sensitive methods, such as multiparametric flow cytometry (MFC) and next-generation sequencing (NGS), still experience disease progression ${ }^{1}$. These bone marrow (BM)-based assessments may lead to false-negative results due to spatial heterogeneity in the BM, hemodilution, and/or extramedullary disease. Positron emission tomography/computed tomography (PET/CT) is complementary but gaps remain ${ }^{2}$.

Peripheral blood (PB)-based methods could potentially overcome these barriers in assessing for residual disease in MM, but time-tested methods such as serum protein electrophoresis (SPEP) and immunofixation (IFIX) that assess for the monoclonal immunoglobulin product in MM do not have sufficient sensitivity (LoD $0.1-0.2 \mathrm{~g} / \mathrm{dL}$ with extended LoD $0.04 \mathrm{~g} / \mathrm{dL}$ if migrate in the gamma fraction) as the monoclonal protein (MP) spike fades into the polyclonal background with lower levels of disease ${ }^{3,4}$. In contrast, mass spectrometry (MS) is able to detect the presence of a plasma cell clone at a heightened sensitivity via the mass/charge ratio of its secreted paraprotein,

\footnotetext{
Correspondence: Andrzej J. Jakubowiak (ajakubowiak@medicine.bsd.uchicago. edu)

${ }^{1}$ Section of Hematology/Oncology, University of Chicago Medical Center,

Chicago, IL, USA

2Department of Hematology and Bone Marrow Transplantation, Poznan

University of Medical Sciences, Poznan, Poland
}

making it uniquely suited to assess for $\mathrm{MRD}^{3,5-7}$. Two prevailing techniques for MS have arisen for analyzing monoclonal immunoglobulins: matrix-assisted laser desorption ionization time-of-flight MS (MALDI-TOF$\mathrm{MS})^{6,8}$ and liquid chromatography (LC) quadrupole timeof-flight $\mathrm{MS}^{5,8}$. While MALDI is a high-throughput method that allows for quick turnaround with LoD $0.05 \mathrm{~g} / \mathrm{dL}$, LC-MS can achieve higher sensitivity with LoD $0.005 \mathrm{~g} / \mathrm{dL}$.

In this study, we assessed the concordance and prognosticative abilities of residual disease status by MS in the PB compared with SPEP/IFIX, PET/CT, and MFC and NGS in the BM to determine if MS may represent a suitable PB-based method for detecting MRD in MM.

Seventy-six patients with newly diagnosed secretory MM were enrolled into a multicenter phase two study investigating the safety and efficacy of four cycles of carfilzomib, lenalidomide, and dexamethasone (KRd) induction followed by high-dose melphalan and autologous stem cell rescue, KRd consolidation for 14 cycles, and lenalidomide maintenance therapy (NCT01816971) ${ }^{9}$. Written informed consent was provided by each individual, and the study was approved by the local Institutional Review Board. Thirty-six patients (characteristics in Table S1) had paired PB and BM samples at the end of cycle 18 KRd (C18) and were included in this analysis; of these patients, 24 also had paired PB and BM samples available at the end of 1 year of lenalidomide maintenance (1yLM). All 36 patients reached a very good partial response or better, and 29 (81\%) achieved a complete response or 
better. MRD by MFC was performed with an LoD between $10^{-4}$ and $10^{-5}$. MRD by NGS in the BM was performed using clonoSEQ (Adaptive Biotechnologies, Seattle, WA) prospectively per protocol with $\mathrm{LoD}<10^{-5}$ based on DNA input ${ }^{10}$. Of the 60 total NGS samples evaluated in this analysis, 18 reached an $\operatorname{LoD}<10^{-6}, 40$ reached an $\mathrm{LoD}<10^{-5}$, and 2 reached an LoD of $10^{-4}-10^{-5}$.

MS was performed by the Binding Site Group using both MALDI-TOF-MS and LC-MS. Analysts performing the MS experiments were blinded to the status of each patient's PB sample (MP isotype and concentration) prior to analysis. The MS methods used have been described previously ${ }^{5,6}$. The unique molecular mass of a monoclonal light chain was defined at baseline and was tracked in each patient's samples ${ }^{5}$. MS results were only considered positive if the original paraprotein at diagnosis was detected.

Concordance between MS by MALDI-TOF and LC-MS in PB, IFIX in PB, PET/CT, MFC in BM, and NGS in BM were assessed using Cohen's kappa $(\kappa)$ statistic, with $\kappa$ value interpretation per Landis and Koch ${ }^{11}$. Progressionfree survival (PFS) curves were constructed using the Kaplan-Meier method and log-rank tests. Hazard ratios (HRs) and 95\% confidence intervals (CIs) for survival data were derived from Cox proportional hazards regression models.

There was substantial concordance $(\kappa=0.667,83 \%$ agreement) between NGS and MALDI-TOF-MS among the 60 samples (Fig. 1A, Fig. S1, and Table S2). Stratified by LoD of the NGS sample (Fig. S2A), the two NGS samples with LoD $10^{-4}-10^{-5}$ were $\mathrm{NGS}^{-} / \mathrm{MALDI}^{-\mathrm{TOF}}{ }^{-}$. There was $80 \%$ agreement $(\kappa=0.591)$ between MALDITOF-MS and NGS with LoD $10^{-5}-10^{-6}$, and $89 \%$ agreement $(\kappa=0.766)$ between MALDI-TOF-MS and NGS with LoD $<10^{-6}$. The only $\mathrm{NGS}^{+} / \mathrm{MALDI}$-TOF$\mathrm{MS}^{-}$case was with an NGS sample with $\mathrm{LoD}<10^{-6}$, and 8/9 (78\%) NGS $^{-} /$MALDI-TOF-MS $^{+}$cases involved NGS samples with LoD between $10^{-5}$ and $10^{-6}$. Three (33\%) of the $\mathrm{NGS}^{-} / \mathrm{MALDI}-\mathrm{TOF}-\mathrm{MS}^{+}$cases clinically progressed, including one patient who converted to $\mathrm{NGS}^{+}$at a later timepoint, but prior to clinical progression.

Concordance between NGS and LC-MS was fair $(\kappa=$ 0.348 ) with $63 \%$ agreement (Table S3 and Fig. S2B). There was no $\mathrm{NGS}^{+} / \mathrm{LC}^{-\mathrm{MS}^{-}}$case compared to $22 \mathrm{NGS}^{-} / \mathrm{LC}-$ $\mathrm{MS}^{+}$cases (from 16 unique patients over the two timepoints; Fig. 1A). Stratified by LoD of the NGS sample, the two NGS samples with LoD $10^{-4}-10^{-5}$ were $\mathrm{NGS}^{-} / \mathrm{LC}$ $\mathrm{MS}^{+}$. There was $60 \%$ agreement $(\kappa=0.309)$ between LCMS and NGS with LoD between $10^{-5}$ and $10^{-6} ; 16 / 40$ (40\%) samples were $\mathrm{NGS}^{-} / \mathrm{LC}_{-} \mathrm{MS}^{+}$. There was $78 \%$ agreement $(\kappa=0.478)$ between LC-MS and NGS with an LoD $<10^{-6}$ with $4 / 18$ (22\%) samples being $\mathrm{NGS}^{-} / \mathrm{LC}$ $\mathrm{MS}^{+}$(Fig. S2B). Of the 16 total patients with discordant

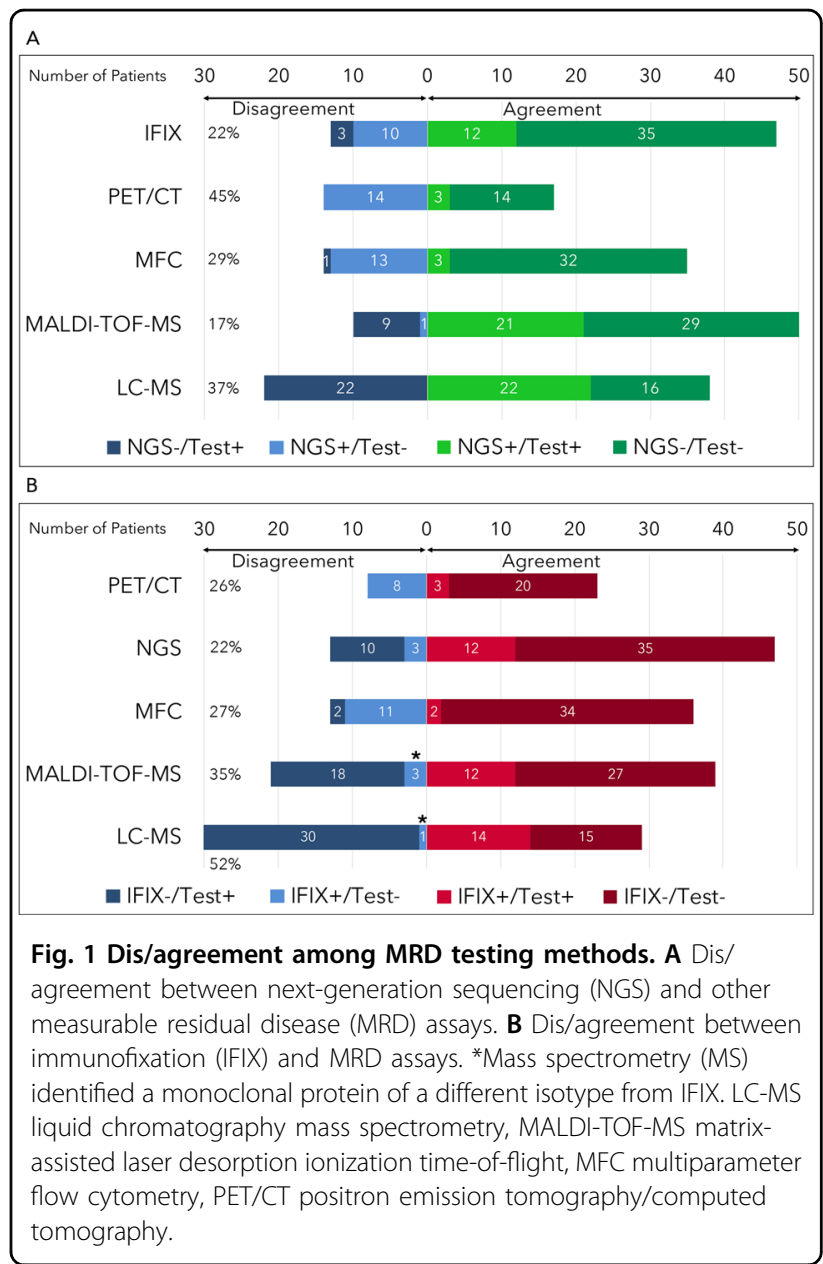

$\mathrm{NGS}^{-} / \mathrm{LC}^{-\mathrm{MS}^{+}}$cases at C18, 5 (31\%) experienced progression, including 3 who converted to $\mathrm{NGS}^{+}$at a later timepoint, but prior to the clinical progression ${ }^{12}$. An example of MALDI-TOF and LC-MS mass spectra of a

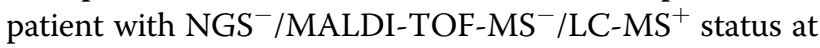
C18 is shown in Fig. S3.

A MP was identified at diagnosis in all 36 patients by both IFIX and MALDI-TOF-MS/LC-MS. There were only three patients who were $\mathrm{IFIX}^{+} / \mathrm{MS}^{-}$(Fig. 1B and Fig. S1); all of these patients were found to have a different clone of the same isotype by MS (i.e., an IgG kappa paraprotein of an entirely different molecular mass from the original), suggesting that IFIX $^{+}$results in these patients did not correspond with the original clone at diagnosis.

MFC (LoD $10^{-4}-10^{-5}$ ) and PET/CT results were available at the same timepoints in 49/60 (82\%) and 31/60 (52\%) samples, respectively. Neither MFC nor PET/CT offered additional discriminatory capacity (Fig. 1 and Fig. S1).

PFS by MRD status at C18 is shown in Fig. 2. With a median follow-up of 56 months, MRD negativity by NGS 


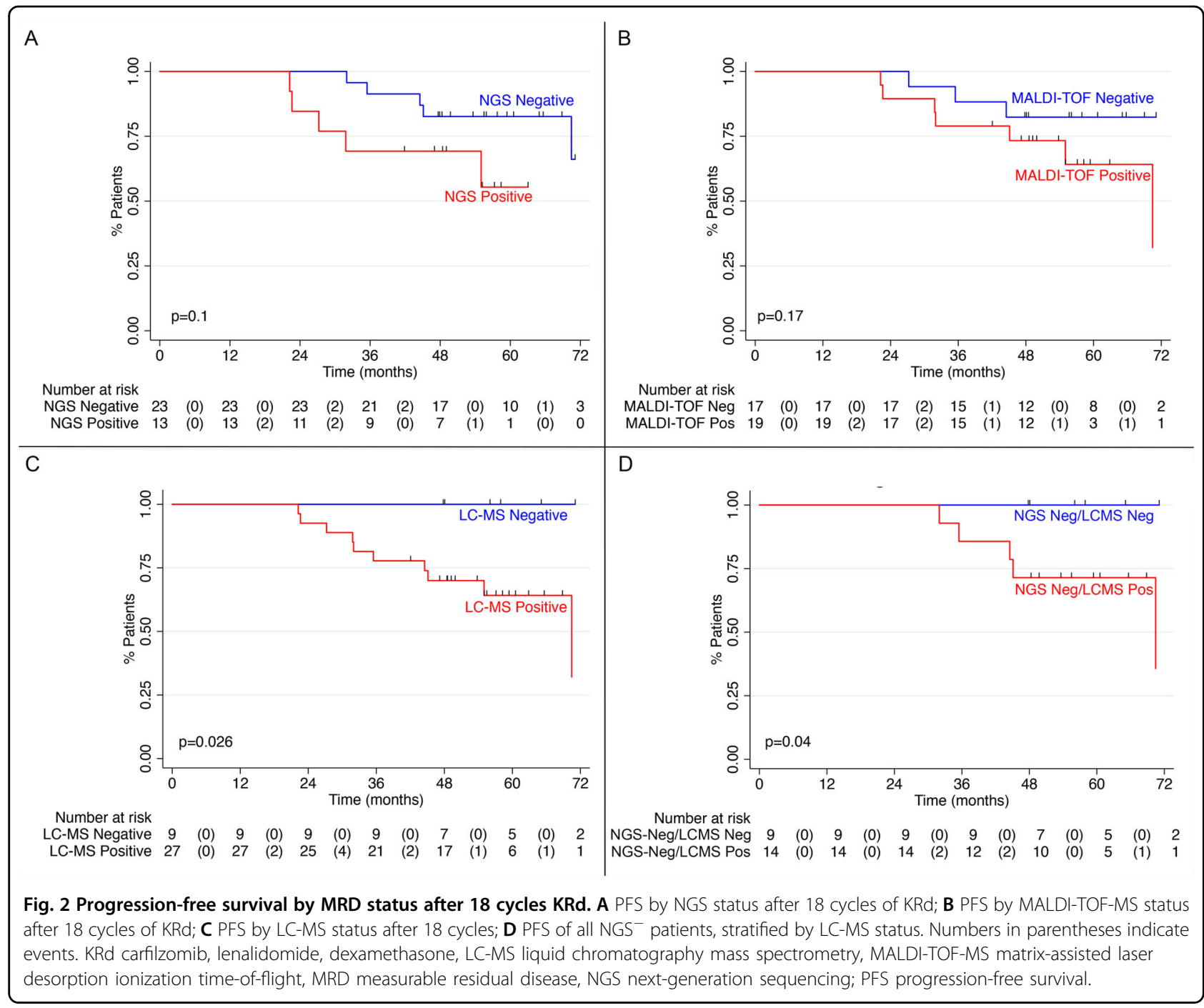

was associated with a nonsignificant PFS benefit (HR 2.9, 95\% CI $0.8-10.8, p=0.11$ ), as was the case for MALDITOF-MS negativity (HR 2.5, 95\% CI 0.6-9.7, $p=0.17$ ). Of the nine patients who were $\mathrm{LC}_{-} \mathrm{MS}^{-}$at $\mathrm{C} 18$, all were alive and free of progression at last follow-up compared to the ten events (including four deaths) in the $\mathrm{C} 18 \mathrm{LC}-\mathrm{MS}^{+}$ group ( $\log$-rank $p=0.026)$. When limiting analysis to only the patients who were MRD negative by NGS, $\mathrm{NGS}^{-} / \mathrm{LC}$ $\mathrm{MS}^{-}(n=9)$ was associated with superior PFS compared to $\mathrm{NGS}^{-} / \mathrm{LC}^{-\mathrm{MS}^{+}}(n=14$, log-rank $p=0.04)$. Comparison of PFS between NGS ${ }^{-}$/MALDI-TOF-MS ${ }^{-}(n=16)$ and NGS ${ }^{-}$MALDI-TOF-MS ${ }^{+}(n=7)$ was limited by a wide $\mathrm{CI}$, though the effect size appeared clinically meaningful (HR 3.4, 95\% CI 0.6-20.1, $p=0.2$ ).

Prior studies have suggested that assessments for malignant plasma cell clones by NGS and next-generation flow (NGF) in the PB have not been able to approximate the LoD of BM testing ${ }^{13,14}$. This is the first study to show that a MS-based PB "liquid biopsy" method for residual disease may be at least as sensitive as BM-based MRD assessments designed to detect one malignant cell per million nucleated cells $\left(\mathrm{LoD} \leq 10^{-6}\right)$ and possibly more sensitive than the current standard of $\operatorname{LoD}<10^{-5}$, suggesting that MS could be considered for MRD detection.

We found that MRD assessment by MALDI-TOF-MS in the PB was at least as sensitive as MRD by NGS with $\mathrm{LoD}<10^{-5}$ in the $\mathrm{BM}$, while LC-MS in the PB was superior to the current standard of MRD negativity with LoD $<10^{-5}$ and at least as sensitive as MRD by NGS with LoD $<10^{-6}$ in the BM. Moreover, LC-MS status appeared to be a superior predictor of PFS compared to NGS or MALDI-TOF-MS status; none of the patients who were $\mathrm{LC}^{-\mathrm{MS}^{-}}$at $\mathrm{C} 18$ experienced progression or death. That a fraction of $\mathrm{LC}^{-\mathrm{MS}^{+}} / \mathrm{NGS}^{-}$patients later converted to $\mathrm{NGS}^{+}$and experienced clinical progression suggests LCMS is a highly sensitive PB technique for detection of MRD that may predict progression sooner than state-ofthe-art BM-based assays. It is unlikely that the $\mathrm{MS}^{+}$cases 
in this study represented false positives as even the earliest PB samples analyzed were nearly 2 years from the start of treatment, when recirculation of existing MP is not expected.

Current evidence suggests that MS in the PB and NGF/ NGS in the BM are complementary methods whose results should be considered as part of a multimodal approach to MRD assessment ${ }^{15}$. Though its use is rising, MS as an assay for MRD is limited to the research setting at the moment, owing to lack of consensus on the appropriate technique (MALDI-TOF-MS vs LC-MS) and availability. It will also be important to factor the cost of these methods into a disease monitoring algorithm. If validated, MS in the PB could be used as a screening method for MRD for patients who have undetectable disease by IFIX and serum free light chains; if MS is negative for disease, a BM biopsy and aspiration could be performed to confirm MRD-negative status. Comprehensive multimodal MRD approaches that incorporate functional imaging with assessments of the $\mathrm{BM}$ and $\mathrm{PB}$ compartments may allow for improved MRD-adaptive clinical trials, and allow for MRD to better guide decisionmaking in MM.

\section{Acknowledgements}

We thank David R. Barnidge of the Binding Site Group, Rochester, MN, USA and Ellen Jenner, Oscar Berlanga, and Stephen Harding of the Binding Site Group Ltd., Birmingham, UK for their assistance in performing the mass spectrometry analyses. This study was presented in abstract form at the 2020 American Society of Clinical Oncology Virtual Conference (abstract \#8513).

\section{Conflict of interest}

B.A.D, A.T.S, K.J, A.M., T.K., and J.K.J, declare no competing financial interests. A.J. J. serves on the advisory boards of Abbvie, Amgen, Celgene, GlaxoSmithKline, Janssen, Juno, Karyopharm, and Sanofi.

\section{Publisher's note}

Springer Nature remains neutral with regard to jurisdictional claims in published maps and institutional affiliations.

Supplementary information The online version contains supplementary material available at https://doi.org/10.1038/s41408-021-00418-2.
Received: 18 September 2020 Revised: 14 December 2020 Accepted: 14 January 2021

Published online: 05 February 2021

\section{References}

1. Perrot, A. et al. Minimal residual disease negativity using deep sequencing is a major prognostic factor in multiple myeloma. Blood 132, 2456-2464 (2018).

2. Moreau, P. et al. Prospective evaluation of magnetic resonance imaging and [18F]fluorodeoxyglucose positron emission tomography-computed tomography at diagnosis and before maintenance therapy in symptomatic patients with multiple myeloma included in the IFM/DFCl 2009 trial: results of the IMAJEM study. J. Clin. Oncol. 35, 2911-2918 (2017).

3. Zajec, M. et al. Mass spectrometry for identification, monitoring, and minimal residual disease detection of M-proteins. Clin. Chem. 66, 421-433 (2020).

4. Murray, D. L. et al. Laboratory persistence and clinical progression of small monoclonal abnormalities. Am. J. Clin. Pathol. 138, 609-613 (2012).

5. Barnidge, D. R. et al. Using mass spectrometry to monitor monoclonal immunoglobulins in patients with a monoclonal gammopathy. J. Proteome Res. 13, 1419-1427 (2014).

6. Mills, J. R. et al. Comprehensive assessment of M-proteins using nanobody enrichment coupled to MALDI-TOF mass spectrometry. Clin. Chem. 62, 1334-1344 (2016)

7. Mills, J. R., Barnidge, D. R., Dispenzieri, A. \& Murray, D. L. High sensitivity bloodbased M-protein detection in SCR patients with multiple myeloma. Blood Cancer J. 7, e590 (2017).

8. Chapman, J. R. \& Thoren, K. L. Tracking of low disease burden in multiple myeloma: Using mass spectrometry assays in peripheral blood. Best Pract. Res. Clin. Haematol. 33, 101142 (2020).

9. Jasielec, J et al. Carfilzomib, lenalidomide, and dexamethasone plus transplant in newly diagnosed multiple myeloma. Blood 136, 2513-2523 (2020).

10. Adaptive Biotechnologies Corporation. clonoSEQ Assay Technical Information. https:/www.clonoseq.com/sites/default/files/ clonoSEQ_TechnicallnformationSummary_21Sept2018.pdf (2010).

11. Landis, J. R. \& Koch, G. G. The measurement of observer agreement for categorical data. Biometrics 33, 159-174 (1977).

12. Kumar, S. et al. International Myeloma Working Group consensus criteria for response and minimal residual disease assessment in multiple myeloma. Lancet Oncol. 17, e328-e346 (2016).

13. Vij, R. et al. Deep sequencing reveals myeloma cells in peripheral blood in majority of multiple myeloma patients. Clin. Lymphoma Myeloma Leuk. 14, 131-139.e1 (2014).

14. Sanoja-Flores, L. et al. Blood monitoring of circulating tumor plasma cells by next generation flow in multiple myeloma after therapy. Blood 134, 2218-2222 (2019)

15. Puíg, N. et al. Analysis of treatment efficacy in the GEM-CESAR trial for high-risk smoldering multiple myeloma patients: comparison between the standard and IMWG MRD criteria and QIP-MS including FLC (QIP-FLC-MS). J. Clin. Oncol. 38, 8512-8512 (2020) 